\title{
Material modelling of FDM printed PLA part
}

\author{
Oleg Volgin ${ }^{\mathrm{a}^{*}}$ and Igor Shishkovsky ${ }^{\mathrm{a}}$
}

\begin{tabular}{|c|c|}
\hline ART I CLE I N F O & A B S T RACT \\
\hline $\begin{array}{l}\text { Article history: } \\
\text { Received } 10 \text { October } 2020 \\
\text { Accepted } 17 \text { December } 2020 \\
\text { Available online } \\
\text { 17 December } 2020 \\
\text { Keywords: } \\
\text { Viscoplasticity } \\
\text { Constitutive model } \\
\text { Material testing }\end{array}$ & $\begin{array}{l}\text { This paper focuses on modelling inelasticity of additively manufactured polylactide (PLA) } \\
\text { thermoplastic using Fused Deposition Modelling (FDM) printing technology. The material response } \\
\text { of PLA is viscoplastic and temperature-dependent, as is typically seen for thermoplastics. The inelastic } \\
\text { deformation of printed PLA undergoes initial yielding, strain softening, and subsequent failure. The } \\
\text { Three-Network (TN) constitutive model was employed in this work, which captures experimentally } \\
\text { observed material response and consists of three molecular equilibrium and time-dependent viscous } \\
\text { networks that act in parallel. The parameter identification was performed in accordance with } \\
\text { experimental data from uniaxial testing and a validation experiment was carried out by loading plate } \\
\text { with a hole and measuring its strain distribution using Digital Image Correlation (DIC) method, which } \\
\text { was compared with the predictions from Finite Element Analysis (FEA). }\end{array}$ \\
\hline
\end{tabular}

\section{Introduction}

Polylactide (PLA) is a relatively inexpensive amorphous thermoplastic possessing high elastic modulus, high tensile strength, low elongation at break and low glass transition temperature $T_{g}$ in the range of $55-65^{\circ} \mathrm{C}$. It is the natural product derived from fermentation of sugars in sugarcane and corn making it eco-friendly and biodegradable under curtain temperature and humidity conditions. Due to its biocompatibility PLA is widely used for many practical applications ranging from plastic cups to medical implants. It can be injected into the human body with minimum inflammation as bone fixation devices, drug delivery systems, ureteral and vascular stents and others (Senatov et al., 2016; Pawar et al. 2014).

Thanks to its low cost and accessibility PLA material is a most common thermoplastic for application in additive manufacturing (AM) meaning construction of a three-dimensional object from a CAD model in a layer-by-layer fashion. This manufacturing process allows producing parts with different geometrical complexities avoiding the use of traditional fabrication techniques. An appropriate 3D printing technology for the fabrication of polymer parts is a fused deposition modelling (FDM) where the melt extrusion method is used to deposit filaments of thermal plastics. However, a critical and common issue of printed parts is that the mechanical characteristics of specimens strongly dependent of printing parameters during processing as temperature, layer thickness, infill percentage and layer stacking

* Corresponding author.

E-mail addresses: oleg.volgin@skoltech.ru (O. Volgin) 
scheme, which determine the fibre-to-fibre bond strength and void density of the parts (Kiendl \& Gao, 2020; Villacres et al. 2018; Chacón et al. 2017; Rajpurohit \& Dave, 2019). Therefore, there is a strong demand to develop advanced material models to predict the mechanical response and failure of FDM polymeric objects. A common approach to analyze FDM materials is to use classical lamination theory considering them as composite laminates (Li et al., 2002; Kulkarni \& Dutta, 1999; Rodríguez et al., 2003; Somireddy et al., 2018), where the validity is restricted to linear elastic analysis. In some previous works, (Ahn et al. 2003; Yao et al., 2020; Zhao et al., 2019; Li et al., 2018; Garzon-Hernandez et al., 2020, Ameri et al., 2020, Vălean et al. 2020 a,b, Bahrami et al. 2020), the authors applied models for predicting the strength and stiffness of PLA and ABS specimens according to the process parameters.

The goal of this work is to apply an isotropic non-linear constitutive model for strain-stress state estimation of PLA material fabricated by FDM technology. The model captures experimentally observed material rate-dependent viscoplastic response and consists of three molecular equilibrium and timedependent viscous networks that act in parallel. The parameter identification was performed in accordance with experimental data from uniaxial testing and a validation experiment was carried out by loading plate with a hole and measuring its strain distribution using DIC method, which was compared with the predictions from FE analysis using Deal.II software.

\section{Constitutive Model}

The Three Network Model (TNM) is a constitutive model for predicting the non-linear timedependent behavior of thermoplastic materials. This model is represented in (Bergström, 2015), and unlike other proposed viscoplastic models TNM shows better numerical efficiency.

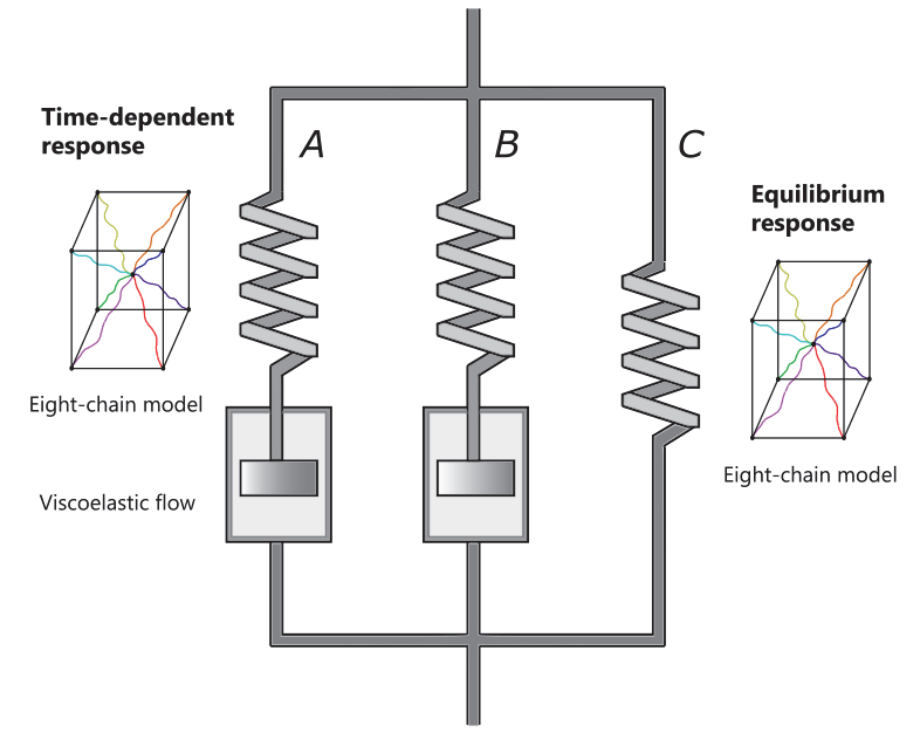

Fig. 1. Rheological representation of the constitutive model.

The behavior of thermoplastic material can be described by mechanical system (Fig. 1), which is composed of three elements connected in parallel. Element $\mathrm{C}$ defines elastic behavior of static loading when the dashpots of elements $\mathrm{A}$ and $\mathrm{B}$ absorb applied deformation, so that springs remain undeformed. Elements A and B, also known as Maxwell elements, perceive stresses under finite deformation rate, and resistance of dampers depends on strain rate. In special case of instant loading dashpots remain closed, and all the springs have to open, thereby increasing stiffness of the system. In literature, it is common to use a frictional device to describe plastic behavior of material, which is not presented in considered rheological model. In order to imitate plastic behavior shear modulus of element B is assumed to evolve with plastic strain.

Deformation of the system is the same as for the individual elements connected in parallel: 


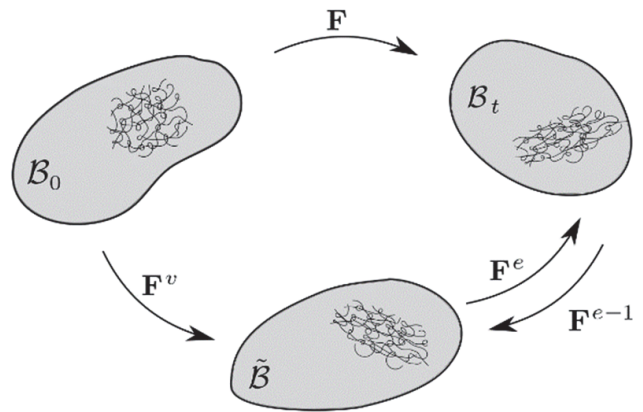

Fig. 2. Kinematics of viscoplastic deformation

The kinematic framework used in the TNM is based on a decomposition of the applied deformation gradient into viscoelastic and viscoplastic components (Fig. 2). Thus, the deformation gradient of element A looks as follows:

$$
\mathbf{F}_{A}=\mathbf{F}_{A}^{e} \mathbf{F}_{A}^{v}
$$

The Cauchy stress of network A for the TNM at a given deformation state is given by the eight-chain model:

$$
\boldsymbol{\sigma}_{A}=\frac{\mu_{A}}{J_{A}^{e} \overline{\lambda_{A}^{e *}}} \frac{\mathcal{L}^{-1}\left(\frac{\overline{\lambda_{A}^{e *}}}{\lambda_{L}}\right)}{\mathcal{L}^{-1}\left(\frac{1}{\lambda_{L}}\right)} \operatorname{dev}\left[\mathbf{b}_{A}^{e *}\right]+\kappa\left(J_{A}^{e}-1\right) \mathbf{I},
$$

where all the necessary parameters depend on the elastic part of the deformation gradient: $J_{A}^{e}=\operatorname{det}\left[\mathbf{F}_{A}^{e}\right]$ is the Jacobian, $\overline{\lambda_{A}^{e *}}=\left(\operatorname{tr}\left[\mathbf{b}_{A}^{e *}\right]\right)^{1 / 2}$ is the effective distortional chain stretch, $\mathbf{b}_{A}^{e *}=\left(J_{A}^{e}\right)^{-2 / 3} \mathbf{F}_{A}^{e}\left(\mathbf{F}_{A}^{e}\right)^{\mathrm{T}}$ is the distortional Cauchy-Green deformation tensor, $\mu_{A}$ is the constant shear modulus, $\lambda_{L}$ is the chain locking stretch and $\kappa$ is the bulk modulus. Similarly to network A, decomposition of the deformation gradient into elastic and viscous parts is applied to element $\mathrm{B}$ :

$$
\mathbf{F}_{B}=\mathbf{F}_{B}^{e} \mathbf{F}_{B}^{v}
$$

As a hyperelastic constitutive law the same eight-chain model is applied to network B:

$$
\boldsymbol{\sigma}_{B}=\frac{\mu_{B}}{J_{B}^{e} \overline{\lambda_{B}^{e *}}} \frac{\mathcal{L}^{-1}\left(\frac{\overline{\lambda_{B}^{e *}}}{\lambda_{L}}\right)}{\mathcal{L}^{-1}\left(\frac{1}{\lambda_{L}}\right)} \operatorname{dev}\left[\mathbf{b}_{B}^{e *}\right]+\kappa\left(J_{B}^{e}-1\right) \mathbf{I}
$$

where $J_{B}^{e}=\operatorname{det}\left[\mathbf{F}_{B}^{e}\right]$ is the Jacobian, $\overline{\lambda_{B}^{e *}}=\left(\operatorname{tr}\left[\mathbf{b}_{B}^{e *}\right]\right)^{1 / 2}$ is the effective distortional chain stretch, $\mathbf{b}_{A}^{e *}=$ $\left(J_{B}^{e}\right)^{-2 / 3} \mathbf{F}_{B}^{e}\left(\mathbf{F}_{B}^{e}\right)^{\mathrm{T}}$ is the distortional Cauchy-Green deformation tensor, $\mu_{B}$ is the shear modulus, which is supposed to evolve from initial value with plastic strain that might appear in the system subjected to sufficiently large deformations. The evolution rate of the shear modulus is proposed to be represented in the following form:

$$
\dot{\mu}_{B}=-\beta\left(\mu_{B}^{i}-\mu_{B}^{f}\right) \dot{\gamma}_{A}
$$

where $\mu_{B}^{f}$ is the final shear modulus of network $\mathrm{B}, \mu_{B}$ is the current shear modulus, and $\dot{\gamma}_{A}$ is the viscoelastic flow rate of network $\mathrm{A}$. Analogously the Cauchy stress for network $\mathrm{C}$ is given by eightchain model:

$$
\boldsymbol{\sigma}_{C}=\frac{\mu_{C}}{J \overline{\lambda^{*}}} \frac{\mathcal{L}^{-1}\left(\frac{\overline{\lambda^{*}}}{\lambda_{L}}\right)}{\mathcal{L}^{-1}\left(\frac{1}{\lambda_{L}}\right)} \operatorname{dev}\left[\mathbf{b}^{*}\right]+\kappa(J-1) \mathbf{I}
$$


where $J=\operatorname{det}[\mathbf{F}]$ is the Jacobian, $\overline{\lambda^{*}}=\left(\operatorname{tr}\left[\mathbf{b}^{*}\right]\right)^{1 / 2}$ is the effective distortional chain stretch, $\mathbf{b}^{*}=$ $J^{-2 / 3} \mathbf{F}(\mathbf{F})^{\mathrm{T}}$ is the distortional Cauchy-Green deformation tensor, $\mu_{C}$ is the constant shear modulus. Finally, the total Cauchy stress of three-network model is given by the summation:

$$
\sigma=\sigma_{A}+\sigma_{B}+\sigma_{C}
$$

The hyperelastic response of the Cauchy stress given in Eq. (1) is based on the eight-chain model and can be calculated for any deformation state: once the internal state $\mathbf{F}^{\boldsymbol{v}}$ of the material is known, the elastic part can be expressed as $\mathbf{F}^{v}=\mathbf{F}\left[\mathbf{F}^{v}\right]^{-1}$. To complete the model the rate of change of the internal state through viscoelastic flow has to be specified. The time derivative of the viscous deformation gradient $\mathbf{F}^{v}$ is present in velocity gradient definition, which is derived for the network A as follows:

$$
\mathbf{L}_{A}=\dot{\mathbf{F}}_{A} \mathbf{F}_{A}^{-1}=\mathbf{L}_{A}^{e}+\mathbf{F}_{A}^{e} \mathbf{L}_{A}^{v}\left(\mathbf{F}_{A}^{e}\right)^{-1}=\mathbf{L}_{A}^{e}+\tilde{\mathbf{L}}_{A}^{v}
$$

where $\mathbf{L}_{A}^{v}=\dot{\mathbf{F}}_{A}^{v}\left(\mathbf{F}_{A}^{v}\right)^{-1}=\mathbf{D}_{A}^{v}+\mathbf{W}_{A}^{v}$ is the inelastic velocity gradient in the current configuration and $\tilde{\mathbf{L}}_{A}^{v}=$ $\widetilde{\mathbf{D}}_{A}^{v}+\widetilde{\mathbf{W}}_{A}^{v}$ is the inelastic velocity gradient in the intermediate configuration $\widetilde{\mathcal{B}}$ (Fig. 1) obtained after elastic unloading by $\mathbf{F}_{A}^{e-1}$. While the rate of shape change $\widetilde{\mathbf{D}}_{A}^{v}$ is described by flow rule, the inelastic spin $\widetilde{\mathbf{W}}_{A}^{v}$ can be prescribed in different ways (Boyce, Weber, \& Parks, 1989). Since an arbitrary rigid body rotation of the relaxed configuration leaves the intermediate configuration stress free, the unloading process from the deformed state is not uniquely defined. It is convenient to set the spin tensor $\widetilde{\mathbf{W}}_{A}^{v}$ to zero. Thus, the following can be obtained:

$$
\tilde{\mathbf{L}}_{A}^{v}=\widetilde{\mathbf{D}}_{A}^{v}=\mathbf{F}_{A}^{e} \dot{\mathbf{F}}_{A}^{v}\left(\mathbf{F}_{A}^{v}\right)^{-1}\left(\mathbf{F}_{A}^{e}\right)^{-1}=\mathbf{F}_{A}^{e} \dot{\mathbf{F}}_{A}^{v} \mathbf{F}^{-1}
$$

The rate of shape change $\widetilde{\mathbf{D}}_{A}^{v}$ can be prescribed in the following form:

$$
\mathbf{D}_{A}^{v}=\dot{\gamma}_{A} \mathbf{N}_{A}
$$

where $\mathbf{N}_{A}$ is a normalised tensor aligned with the deviatoric driving stress state:

$$
\mathbf{N}_{A}=\frac{\operatorname{dev}\left[\boldsymbol{\sigma}_{A}\right]}{\left\|\operatorname{dev}\left[\boldsymbol{\sigma}_{A}\right]\right\|}=\frac{\operatorname{dev}\left[\boldsymbol{\sigma}_{A}\right]}{\tau_{A}}
$$

The deviatoric flow rate $\dot{\gamma}_{A}$ is given by the reptation-inspired equation :

$$
\dot{\gamma}_{A}=\dot{\gamma}_{0}\left(\frac{\tau_{A}}{\hat{\tau}_{A}+a R\left(p_{A}\right)}\right)^{m_{A}}
$$

where $\hat{\tau}_{A}, a, m_{A}$ are the material parameters, $p_{A}=\operatorname{tr}\left[\sigma_{A}\right] / 3$ is the hydrostatic pressure, $R(x)=$ $(x+|x|) / 2$ is the ramp function, and $\dot{\gamma}_{0}=1 / s$ is a constant parameter to satisfy dimensional consistency of the equation. The velocity gradient of the viscoelastic flow of network A expressed from Eq. (10) is given as follows:

$$
\dot{\mathbf{F}}_{A}^{v}=\dot{\gamma}_{A} \mathbf{F}_{A}^{e-1} \frac{\operatorname{dev}\left[\boldsymbol{\sigma}_{A}\right]}{\tau_{A}} \mathbf{F}
$$

Analogously, the deviatoric flow rate $\dot{\gamma}_{B}$ can be written:

$$
\dot{\gamma}_{B}=\dot{\gamma}_{0}\left(\frac{\tau_{B}}{\hat{\tau}_{B}+a R\left(p_{B}\right)}\right)^{m_{B}}
$$

where $\hat{\tau}_{A}, m_{A}$ are the material parameters, $p_{B}=\operatorname{tr}\left[\sigma_{B}\right] / 3$ is the hydrostatic pressure. Thus, the velocity gradient of the viscoelatic flow of network $\mathrm{B}$ can be written: 


$$
\dot{\mathbf{F}}_{B}^{v}=\dot{\gamma}_{B} \mathbf{F}_{B}^{e-1} \frac{\operatorname{dev}\left[\boldsymbol{\sigma}_{B}\right]}{\tau_{B}} \mathbf{F}
$$

\section{Materials and Methods}

The material was tested in uniaxial tension using Instron 5969 testing system. The uniaxial tension tests were performed on dogbone-shaped specimens with a diameter of $10 \mathrm{~mm}$ in the gauge section and a gauge length of $25 \mathrm{~mm}$. The tension specimens were deformed in displacement control at different rates to final failure from $0.5 \mathrm{~mm} / \mathrm{min}$ to $50 \mathrm{~mm} / \mathrm{min}$. All tests were performed at room temperature on 5 specimens for each strain-rate and the median stress-strain experimental curves were presented. The results from the uniaxial testing are summarized in Fig. 3. This figure shows that PLA is almost linear elastic for strains less than 0.01 , and then starts to undergo yielding and softening until the final break at 0.05 strain. The figures also show that the material is relatively highly strain-rate dependent.

All testing specimens from PLA were processed using Picaso Designer X Pro with bed temperature $60^{\circ} \mathrm{C}$ and extrusion temperature $220^{\circ} \mathrm{C}$. STL models were oriented horizontally on the building platform with [45/-45] layups, $0.2 \mathrm{~mm}$ layer thickness and 100\% infill percentage with two contour fibers.

In addition to the experimental tests of ordinary specimens, the plate with dimensions $60 \mathrm{~mm} \times 30$ $\mathrm{mm} \times 8 \mathrm{~mm}$ with a round hole in the middle and $5 \mathrm{~mm}$ in diameter was subjected to uniaxial tension with strain rate $1 \mathrm{~mm} / \mathrm{min}$ at the room temperature. A non-contact video extensometer was used to track the strain distribution in force direction, which was later compared with the FE simulation.

\section{Results and Discussion}

The TNM material model was calibrated to the uniaxial experimental data. Parameter $a$ was set to 0, since the specimens were subjected to uniaxial tension. The exponentials $m_{A}$ and $m_{B}$ are chosen to be less than 20 to ensure proper convergence, and the flow resistance $\hat{\tau}_{A}$ has been chosen to be less than $\hat{\tau}_{B}$. The parameter set was optimized using the least squares method and providing a set of initial guesses. During calibration the transverse strains have to be calculated providing zero stresses in transverse directions. This optimization problem can be solved by the derivative-free Nelder-Mead method (downhill simplex algorithm) used in Nelder \& Mead (1965). Solving the minimisation problem requires varying of the stretches in lateral directions, whereas the stretch in longitudinal direction remains fixed during the step, which means that the differential Eq.(6), Eq. (14) and Eq. (16) have to be solved at each iteration step. Assuming that viscous part of the deformation gradient $\mathbf{F}_{n}^{v}$ is a known quantity from the previous time step, entries of the $\mathbf{F}_{n+1}^{v}$ at the next time step can be approximated. The resulting material parameters are listed in Table 1.

Table 1. Material parameters used by the three-network model

\begin{tabular}{ccc}
\hline Symbols & Values & Description \\
\hline$\mu_{A}$ & 315.74 & Shear modulus of network $A$ \\
$\lambda_{L}$ & 16.05 & Locking stretch \\
$\kappa$ & 500 & Bulk modulus \\
$\hat{\tau}_{A}$ & 22.29 & Flow resistance of network $A$ \\
$a$ & 0 & Pressure dependence of flow \\
$m_{A}$ & 15 & Stress exponential of network $A$ \\
$\mu_{B}^{i}$ & 862.4 & Initial shear modulus of network $B$ \\
$\mu_{B}^{f}$ & 141.64 & Final shear modulus of network $B$ \\
$\beta$ & 66.74 & Evolution rate of $\mu_{B}$ \\
$\hat{\tau}_{B}$ & 49.72 & Flow resistance of network $B$ \\
$m_{B}$ & 8.1 & Stress exponential of network $B$ \\
$\mu_{C}$ & 2.5 & Shear modulus of network $C$ \\
\hline
\end{tabular}


A direct comparison between the experimental data and the model predictions is shown in Fig. 3 . This figure demonstrates that the calibrated material model is in good agreement with the experimental results and captures the yield evolution as well as the strain-rate dependence of the material.

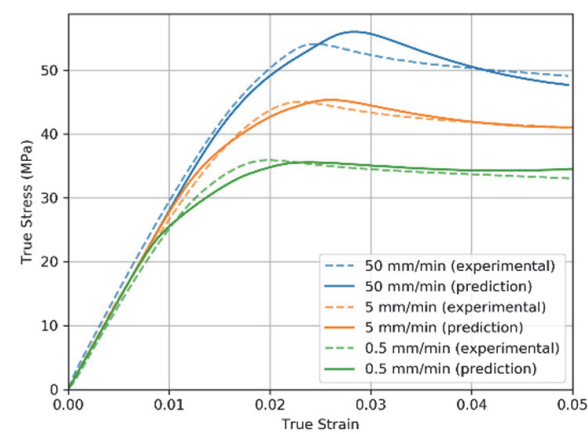

Fig. 3. Stress-strain behavior of PLA at room temperature
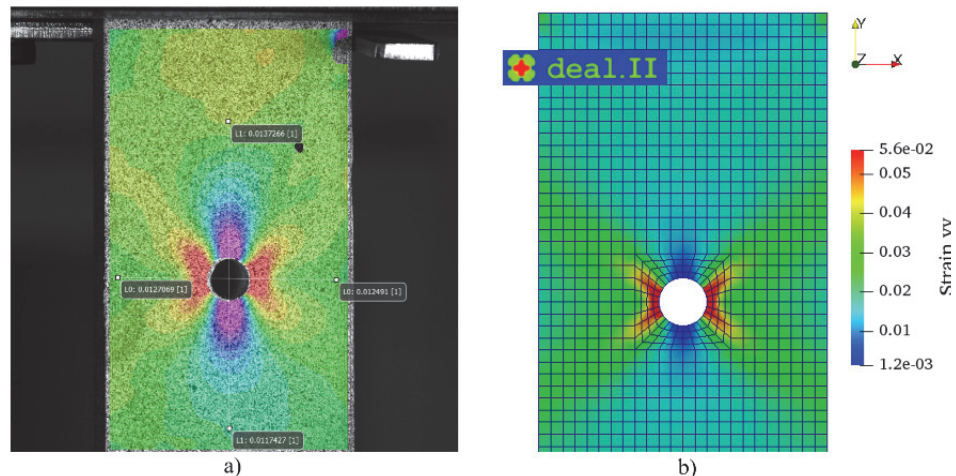

Fig. 4. (a) DIC and (b) FEA results of $e_{y y}$ at $1.2 \mathrm{~mm}$ elongation

To validate the material model it was implemented in the FEM framework into a Deal.II environment. Finite element model of the plate tension test with a concentrator was investigated. The results from the experiment and simulation are shown in Fig. 4 at $1.2 \mathrm{~mm}$ elongation before cracking that considerably altered the symmetric stress-strain state of the plate with further loading. This figure illustrates that the calibrated material model accurately predicts the strain distribution response of the test. Y-directional strains are dominant during uniaxial tension, thus the model has the same von Mises stress distribution pattern (Fig. 5b), which is proved by diagonal $45^{\circ}$ angle fraction behavior of the sample.
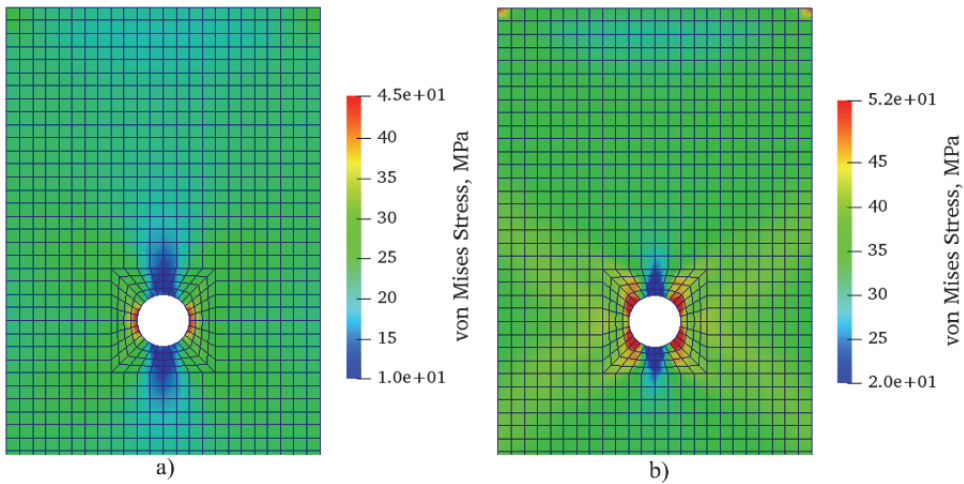

Fig. 5. FEA stress estimations at (a) $0.5 \mathrm{~mm}$ and (b) $1.2 \mathrm{~mm}$ elongations

Experimental and calculated y-displacements were presented and compared in Fig. 6a. The values between the diagrams differ slightly, however, the experimental diagram is qualitatively nonlinear in comparison with the calculated one, which rather exhibits linear behavior. This can be explained by inaccuracies in calculations. The stress diagram is presented in Fig. 6b, which demonstrates that calculated maximum von Mises stresses appearing in the plate are in good agreement with experimental data, since the ultimate stress right before breaking of the specimen was achieved. The behavior of the specimen is elastic until it starts to yield at $0.5 \mathrm{~mm}$ elongation and its stress distribution was compared in Fig. 5 with the final stress state, which corresponds to $1.2 \mathrm{~mm}$. It can be seen from the figure that the stress distribution pattern is non-constant during simulation due to the softening effect in concentrated areas and making the neighboring areas to undergo higher stresses with further elongation. It can be proved by analytical thinking in accordance with the maximum force $9.3 \mathrm{kN}$ applied in the experiment 
and the weakest cross-sectional area of the plate $200 \mathrm{~mm}^{2}$ resulting in $46,5 \mathrm{MPa}$ average stress, which corresponds to the FE result shown in Fig. 5b.

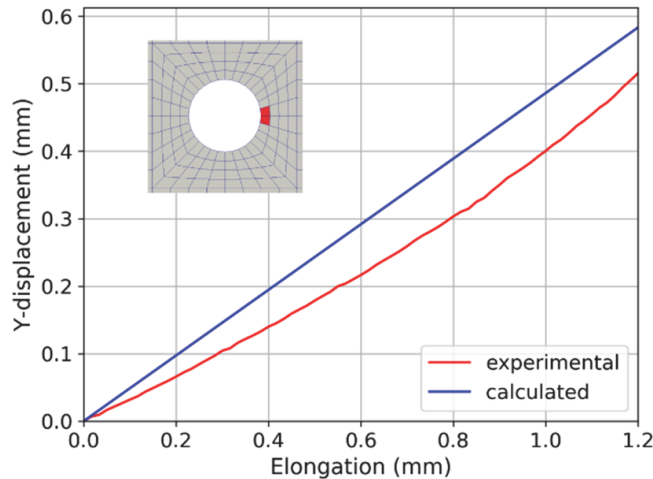

a)

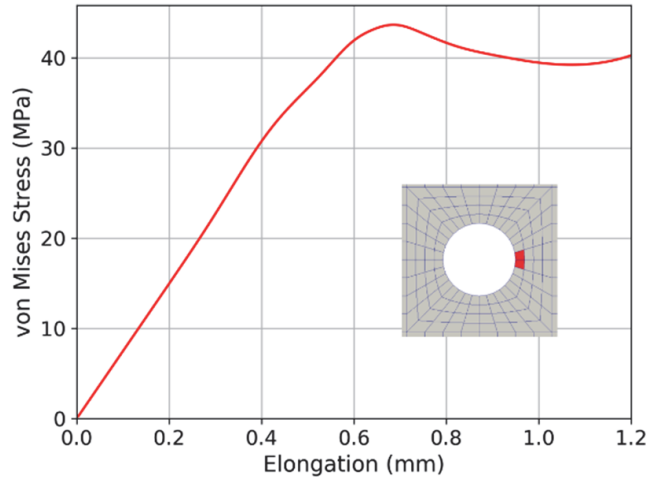

b)

Fig. 6. Experimental and predicted y-displacements (a) and von-Mises stress estimation (b) at the area indicated in red

\section{Conclusion}

An isotropic material model that captures the viscoplastic material behavior of FDM printed PLA parts subjected to the uniaxial loading was validated. The parallel network material model was calibrated to the experimental data. The model predictions were then also validated during the tension experiment of plate with concentrator and the results showed favorable comparison between FE model and experiment. The current work shows that it is possible to predict the mechanical response of FDM material by application of the inelastic material model and identification of its parameter set. There are still many open problems awaiting investigation in the future. Further mechanical testing should be carried out to gain a deeper understanding of the mechanical behavior of FDM printed PLA as well as developing an anisotropic constitutive model for non-uniaxial loading cases. Due to lammelar structure of printed objects the transverse isotropy could be considered in order to account for printing direction. In addition, thermo-mechanical coupling and phase transition from glassy state to rubbery state could be further incorporated into the model in future. There might be also considered the influence of printing parameters and filling pattern on material behavior.

\section{References}

Ahn, S. H., Baek, C., Lee, S., \& Ahn, I. S. (2003). Anisotropic tensile failure model of rapid prototyping parts - Fused Deposition Modeling (FDM). International Journal of Modern Physics B, 17, 15101516.

Ameri, B., Taheri-Behrooz, F., \& Aliha, M. R. M. (2020). Fracture loads prediction of the modified 3Dprinted ABS specimens under mixed-mode I/II loading. Engineering Fracture Mechanics, 235, 107181.

Bahrami, B., Ayatollahi, M. R., Sedighi, I., Pérez, M. A., \& Garcia-Granada, A. A. (2020). The effect of in-plane layer orientation on mixed-mode I-II fracture behavior of 3D-printed poly-carbonate specimens. Engineering Fracture Mechanics, 107018.

Bergström, J. S. (2015). Mechanics of Solid Polymers. Chadds Ford, PA, USA: FluoroConsultants Group.

Boyce, M. C., Weber, G. G., \& Parks, D. M. (1989). On the kinematics of finite strain plasticity. Journal of the Mechanics and Physics of Solids, 37, 647-665.

Chacón, J. M., Caminero, M. A., García-Plaza, E., \& Núñez, P. J. (2017). Additive manufacturing of PLA structures using fused deposition modelling: Effect of process parameters on mechanical properties and their optimal selection. Materials and Design, 124, 143-157. 
Garzon-Hernandez, S., Garcia-Gonzales, D., Jérusalem, A., \& Arias, A. (2020). Design of FDM 3D printed polymers: An experimental-modelling methodology for the prediction of mechanical properties. Materials and Design, 188, https://doi.org/10.1016/j.matdes.2019.108414.

Kiendl, J., \& Gao, C. (2020). Controlling toughness and strength of FDM 3D-printed PLA components through the raster layup. Composites Part B: Engineering, 180,

Kulkarni, P., \& Dutta, D. (1999). Deposition Strategies and Resulting Part Stiffnesses in Fused Deposition Modeling. Journal of Manufacturing Science and Engineering, 121, 93-103.

Li, H., Wang, T., Sun, J., \& Yu, Z. (2018). The effect of process parameters in fused deposition modelling on bonding degree and mechanical properties. Rapid Prototyping Journal, 24, 80-92.

Li, L., Sun, Q., Bellehumeur, C., \& Gu, P. (2002). Composite Modeling and Analysis for Fabrication of FDM Prototypes with Locally Controlled Properties. Journal of Manufacturing Processes, 4, 129141.

Nelder, J. A., \& Mead, R. (1965). A Simplex Method for Function Minimization. The Computer Journal, 7, 308-313.

Pawar, R. P., Tekale, S. U., Shisodia, S. U., Totre, J. T., \& Domb, A. J. (2014). Biomedical Applications of Poly(Lactic Acid). Recent Patents on Regenerative Medicine (Discontinued), 4, 40-51.

Rajpurohit, S., \& Dave, H. (2019). Analysis of tensile strength of a fused filament fabricated PLA part using an open-source 3D printer. The International Journal of Advanced Manufacturing Technology, $101,1525-1536$.

Rodríguez, J. F., Thomas, J. P., \& Renaud, J. E. (2003). Mechanical behavior of acrylonitrile butadiene styrene fused deposition materials modeling. Rapid Prototyping Journal, 9, 219-230.

Senatov, F. S., Niaza, K. V., Zadorozhnyy, M. Y., Maksimkin, A. V., Kaloshkin, S. D., \& Estrin, Y. Z. (2016). Mechanical properties and shape memory effect of 3D-printed PLA-based porous scaffolds. Journal of the Mechanical Behavior of Biomedical Materials, 57, 139-148.

Somireddy, M., Czekanski, A., \& Singh, C. V. (2018). Development of constitutive material model of 3D printed structure via FDM. Materials Today Communications, 15, 143-152.

Vălean, C., Marșavina, L., Mărghitaș, M., Linul, E., Razavi, J., \& Berto, F. (2020a). Effect of manufacturing parameters on tensile properties of FDM printed specimens. Procedia Structural Integrity, 26, 313-320.

Vălean, C., Marșavina, L., Mărghitaș, M., Linul, E., Razavi, J., Berto, F., \& Brighenti, R. (2020b). The effect of crack insertion for FDM printed PLA materials on Mode I and Mode II fracture toughness. Procedia Structural Integrity, 28, 1134-1139.

Villacres, J., Nobes, D., \& Ayranci, C. (2018). Additive manufacturing of shape memory polymers: effects of print orientation and infill percentage on mechanical properties. Rapid Prototyping Journal, 24, 744-751.

Yao, T., Ye, J., Deng, Z., Zhang, K., Ma, Y., \& Ouyang, H. (2020). Tensile failure strength and separation angle of FDM 3D printing PLA material: Experimental and theoretical analyses. Composites Part B: Engineering, 188,

Zhao, Y., Chen, Y., \& Zhou, Y. (2019). Novel mechanical models of tensile strength and elastic property of FDM AM PLA materials: Experimental and theoretical analyses. Materials and Design, 181.

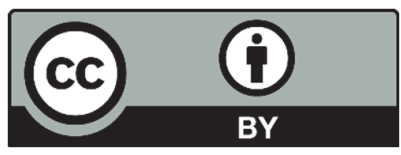

(C) 2021 by the authors; licensee Growing Science, Canada. This is an open access article distributed under the terms and conditions of the Creative Commons Attribution (CC-BY) license (http://creativecommons.org/licenses/by/4.0/). 